\title{
Interface-Driven Phenomena in Solids: Thermodynamics, Kinetics and Chemistry
}

\author{
FADI ABDELJAWAD ${ }^{1,2}$ and STEPHEN M. FOILES ${ }^{1}$ \\ 1.-Materials Science and Engineering Center, Sandia National Laboratories, Albuquerque, \\ NM 87185, USA. 2.—e-mail: fabdelj@sandia.gov
}

The study of materials interfaces dates back over a century. ${ }^{1-3}$ In solid systems and from an engineering perspective, free surfaces and internal (grain and/or phase) boundaries influence a wide range of properties, such as thermal, electrical and optical transport, ${ }^{4-7}$ and mechanical ones. ${ }^{8}$ The properties and the role of interfaces has been discussed extensively in various reviews such as by Sutton and Balluffi. ${ }^{9}$ As the characteristic feature size of a materials system (i.e., grain size) is decreased to the nanometer scale, interface-driven physics is expected to dominate due to the increased density of such planar defects. ${ }^{10-12}$ Moreover, interfacial attributes, thermodynamics, and mobility play a key role in phase transformations, such as solidification dynamics and structural transitions in solids, ${ }^{13-15}$ and in homogenization and microstructural evolution processes, such as grain growth, ${ }^{14}$ coarsening, ${ }^{16}$ and recrystallization. ${ }^{17,18}$

Despite the long history of research into the properties and influence of interfaces, this important topic continues to attract significant attention. One thread of current interest transitions the descriptions of interfaces from simple treatments in terms of average or typical interface properties to ones that reflect the variability of interface properties with respect to interface crystallography, temperature, impurities and alloying. For example, there has recently been extensive work on understanding the variation of grain boundary energy ${ }^{19}$ and mobility ${ }^{20}$ with boundary crystallography. There is ongoing interest in the interaction of interfaces with other materials defects such as impurities and dislocations as well as the behavior of defects within interfaces such as steps, kinks and facets. Another trend is a focus on the unique features of interfaces related to

Fadi Abdeljawad and Stephen Foiles are the JOM advisors for the Chemistry \& Physics of Materials Committee, a joint committee of the TMS Functional Materials Division (FMD) and the TMS Structural Metals Division (SMD); and guest editors for the topic Interface-Driven Phenomena in Solids: Thermodynamics, Kinetics, and Chemistry in this issue. multicomponent systems including heterophase interfaces and interfacial segregation. The collection of articles presented here describes and reviews recent findings of materials phenomena that are greatly influenced by interfaces and their interaction with other defects/features in these systems. This collection of manuscripts is not inclusive of the entire complex yet interesting interface-driven phenomena in materials science, but provides a sampling of the diverse technical challenges associated with interfacial related materials.

In an article entitled "Zener Pinning of Grain Boundaries and Structural Stability of Immiscible Alloys", Koju et al. report an atomistic study of the thermal stability and retardation of grain growth dynamics of immiscible $\mathrm{Cu}-\mathrm{Ta}$ binary alloys. Their study of grain boundary (GB)-alloy interactions under various compositions and GB velocities (via the use of shear-coupled GBs and the externally imposed shear strain) revealed that the experimentally observed stability of these alloys is primarily due to Ta nano-clusters that are coherent with the $\mathrm{Cu}$ matrix, which provide a pinning mechanism found to be in quantitative agreement with the Zener model of GB pinning.

Coleman et al. discuss the experimental and computational hurdles to the understanding the role of grain boundaries in ceramics in the article "Challenges of Engineering Grain Boundaries in Boron-based Armor Ceramics". This paper focuses on boron carbide and boron suboxide ceramics. Experimental challenges focus on the processing of these materials to obtain full density while controlling the microstructure. Computationally, the study of internal interfaces in these materials is complicated by the complex crystal structures. The authors point to the need to combine computations with experiments in order to advance the engineering of this class of materials.

The article "Atomic-Scale Studies of Defect Interactions with Homo- and Heterophase Interfaces" by Martinez et al. examines, via atomistic simulations, 
the interaction of interfaces with other defects, which are typically encountered in mechanical and irradiation environments. They employed two types of semi-coherent interfaces, a face-centered cubicbody-centered cubic (fcc-bcc) $\mathrm{Cu}-\mathrm{Nb}$ interface and an asymmetric $\Sigma 11 \mathrm{~GB}$ in $\mathrm{Cu}$, and compared their response under various scenarios. Interface-dislocation interactions were examined in mechanically deformed systems, where dislocation absorption, transmission, reflection and/or nucleation depend greatly on the local interface atomic structure and intrinsic defect content. Next, the authors examined the interaction of the same interfaces with extended defects, stacking fault tetrahedral and self-interstitial clusters, and revealed that these interactions play a major role in removing irradiation-created defects and dictate the interface sink strength.

In the final paper of this collection, Clark et al. in "Thermal Stability Comparison of Nanocrystalline Fe-Based Binary Alloy Pairs" examined GB solute segregation in nanocrystalline binary alloys and its impact on the thermal stability of such systems. In their study, the authors examined two Fe-based alloy systems, $\mathrm{Fe}-\mathrm{Mg}$ and $\mathrm{Fe}-\mathrm{Cu}$, which were prepared, processed and characterized using the same methodologies. It was shown that the Fe-Mg system exhibited improved thermal stability over the $\mathrm{Fe}-\mathrm{Cu}$ one. Chemical mapping analysis showed that the $\mathrm{Fe}-\mathrm{Mg}$ system is characterized by GBs that are rich in $\mathrm{Mg}$, while the $\mathrm{Fe}-\mathrm{Cu}$ showed a strong tendency for $\mathrm{Cu}$ bulk phase separation. Results from Monte Carlo alloy simulations showed microstructures that are consistent with the experimental observations of both the $\mathrm{Fe}-\mathrm{Mg}$ and $\mathrm{Fe}-\mathrm{Cu}$ systems.

In summary, the set of articles published in this special topic titled: "Interface-Driven Phenomena in Solids: Thermodynamics, Kinetics and Chemistry" covers topics related to microstructure evolution, segregation/adsorption phenomena and interface interactions with other materials defects. We recognize that this collection of articles is a mere drop in an ocean in terms of the wide range of materials phenomena that are greatly influenced by interfaces but hope that readers find them interesting and thought provoking. To download any of the papers, follow the url http://link.springer.com/journal/ $11837 / 68 / 6 /$ page/ 1 to the table of contents page for the June 2016 issue (vol. 68, no. 6).

- "Zener Pinning of Grain Boundaries and Structural Stability of Immiscible Alloys" by R.K. Koju, K.A. Darling, L. Kecskes, and Y. Mishin.

- "Challenges of Engineering Grain Boundaries in
Boron-Based Armor Ceramics" by Shawn P. Coleman, Efrain Hernandez-Rivera, Kristopher D. Behler, Jennifer Synowczynski-Dunn, and Mark A. Tschopp.

- "Atomic-Scale Studies of Defect Interactions with Homo- and Heterophase Interfaces" by Enrique Martinez, Blas P. Uberuaga, and Irene J. Beyerlein.

- "Thermal Stability Comparison of Nanocrystalline Fe-Based Binary Alloy Pairs” by B.G. Clark, K. Hattar, M.T. Marshall, T. Chookajorn, B.L. Boyce, and C.A. Schuh.

\section{ACKNOWLEDGEMENTS}

We acknowledge support by the U.S. Department of Energy, Office of Basic Energy Sciences, Division of Materials Sciences and Engineering. Sandia is a multi-program laboratory operated by the Sandia Corporation, a Lockheed Martin Company, for the United States Department of Energy's National Nuclear Security Administration under Contract DE-AC04-94AL85000.

\section{REFERENCES}

1. P. Curie, Bull. Min. France 8, 145 (1885).

2. J.W. Gibbs, Trans. Conn. Acad. 3, 343 (1878).

3. G. Wulff, Z. Kristallogr. 34, 449 (1901).

4. D.G. Cahill, W.K. Ford, K.E. Goodson, G.D. Mahan, A. Majumdar, H.J. Maris, R. Merlin, and S.R. Phillpot, J. Appl. Phys. 93, 793 (2003).

5. R.J. Gehr and R.W. Boyd, Chem. Mater. 8, 1807 (1996).

6. L. Lu, Y.F. Shen, X.H. Chen, L.H. Qian, and K. Lu, Science 304, 422 (2004).

7. D.L. Medlin and G.J. Snyder, Curr. Opin. Colloid Interface Sci. 14, 226 (2009).

8. K. Lu, L. Lu, and S. Suresh, Science 324, 349 (2009).

9. A.P. Sutton and R.W. Balluffi, Interfaces in Crystalline Materials (Oxford: Clarendon Press, 1995).

10. E. Arzt, Acta Mater. 46, 5611 (1998).

11. M.A. Meyers, A. Mishra, and D.J. Benson, Prog. Mater Sci. 51,427 (2006).

12. J.R. Weertman, D. Farkas, K. Hemker, H. Kung, M. Mayo, R. Mitra, and H. Van Swygenhoven, MRS Bull. 24, 44 (1999).

13. J.S. Langer, Rev. Mod. Phys. 52, 1 (1980).

14. W.W. Mullins, J. Appl. Phys. 27, 900 (1956).

15. A.G. Khachaturyan, Theory of Structural Transformations in Solids (New York: Dover, 2008).

16. L. Ratke and P.W. Voorhees, Growth and Coarsening: Ripening in Material Processing (Berlin: Springer, 2002).

17. F.J. Humphreys and M. Hatherly, Recrystallization and Related Annealing Phenomena, 2nd ed. (Amsterdam: Elsevier, 2004).

18. G. Gottstein and L.S. Shvindlerman, Grain Boundary Migration in Metals: Thermodynamics, Kinetics, and Applications, 2nd ed. (Boca Raton: CRC Press, 2009).

19. V.V. Bulatov, B.W. Reed, and M. Kumar, Acta Mater. 65, 161 (2014).

20. E.R. Homer, E.A. Holm, S.M. Foiles, and D.L. Olmsted, JOM 66, 114 (2014). 